\title{
Bienvenue à Bâle: maladies chroniques - un défi global
}

\section{Nino Künzli ${ }^{a}$}

Nicole Probst-Hensch ${ }^{b}$

a Prof. Dr méd. et Ph.D., professeur ordinaire d'une chaire de médecine sociale et préventive auprès de l'Université de Bâle et vicedirecteur de l'Institut

Tropical et de Santé Publique Suisse Swiss TPH

b Prof. Dr phil. et Ph.D., professeur extraordinaria de santé publique auprès de l'Université de Bâle, directrice du département d'épidémiologie consacré aux maladies chroniques, vicedirectrice du département épidémiologie et santé publique au sein du Swiss TPH

\section{Correspondance:}

Prof. Dr Nino Künzli

Institut Tropical et de Santé

Publique Suisse

Socinstrasse 57

Case postale

CH-4052 Bâle

nino.kuenzli@unibas.ch
Bâle sera fin août 2011 sous le signe des maladies chroniques - l'atelier sur le thème international hautement actuel «Etudes de méga-cohortes» (24 août 2011) sera suivi de la conférence annuelle de Santé Publique Suisse (25/26 août 2011), laquelle se penchera sur le thème phare avec son débat public «Ville saine - une contradiction?»

La conférence au sommet des Nations Unies sur les maladies non transmissibles [1] se tiendra à New York dès le mois de septembre. Une visée essentielle de cette conférence est l'adoption d'un objectif de baisse de $2 \%$ par an des taux de mortalité dus aux maladies chroniques. Les maladies non transmissibles représentent aujourd'hui dans le monde les plus importantes causes de décès. Des organisations telles que l'OMS ou la Banque mondiale mettent en garde contre les conséquences sanitaires et économiques de l'épidémie globale de maladies chroniques.

Quelques exemples: en Suisse, au moins 4\% de la population vit avec le diabète mellitus - c'est une estimation, le chiffre exact est inconnu. Dans les régions africaines au sud du Sahara - avec un état des données encore plus désolant - on pense que dans certains endroits jusqu'à 16\% de la population souffrent du diabète. D'après des estimations encore, env. 2 nouveaux cas de diabète apparaissent chaque année pour 1000 habitants en Inde. En Suisse, le cancer est la cause de décès la plus répandue dans la tranche des 45-64 ans. Le Programme national contre le cancer 2011-2015 nouvellement paru souligne que le taux d'incidence du cancer continue d'augmenter. Pour la première fois, nous disposons également pour la Suisse de chiffres relatifs à l'incidence de la bronchopneumopathie chronique obstructive: d'après l'étude SAPALDIA, on compte env. 14 nouveaux cas par an pour 1000 habitants.

L'allongement de la durée de vie n'explique que partiellement l'épidémie de maladies chroniques. Il existe donc un grand potentiel dans le domaine de la prévention. Des causes essentielles de ces maladies se trouvent dans les conséquences de l'urbanisation de notre espace de vie. Plus de la moitié de la population mondiale vit aujourd'hui dans un environnement ur-

Santé publique Suisse organise la Conférence Suisse de Santé Publique conjointement avec I'Institut Tropical et de Santé Publique Suisse. En tant qu'institution académique de santé publique la plus importante de Suisse, ce dernier défend la santé de la population en s'engageant dans la recherche, la prestation de services et l'enseignement. Santé publique Suisse défend, en tant qu'organisation faîtière nationale, les intérêts de la santé publique: elle cherche à renforcer et à développer ce champ. Elle souhaite également optimiser son application sur le terrain. Informations concernant les manifestations: www.public-health.ch; www.swisstph.ch

bain. Cela implique de fortes modifications du style de vie, de l'environnement ainsi que de l'entourage social et économique. Les substances nocives issues de la circulation, de l'industrie et des ménages affectent l'air, l'eau et les sols. Même dans les pays du Sud et de l'Est, les influences de la télévision, de la publicité et du marketing contribuent de manière décisive au bouleversement social. Les maladies chroniques n'y remplacent pas les maladies transmissibles largement répandues, mais au contraire viennent s'ajouter aux problèmes déjà existants. Les systèmes de santé de ces pays doivent ainsi faire face à d'immenses défis.

Aussi bien dans les pays du Sud que du Nord, la recherche forme un pilier central permettant de mettre en place de façon efficace des stratégies fondées sur les faits en vue de la prévention et du traitement des maladies chroniques. Les études de longue durée et de grande envergue constituent le noyau de l'exploration des maladies chroniques. C'est pourquoi l'Institut Tropical et de Santé Publique Suisse organise un atelier d'une journée voué à la recherche sur les cohortes. Des experts suisses et étrangers présenteront comment des cohortes avec plusieurs milliers de participants sont mises en place et maintenues durant des années. Des débats sur les objectifs, les contenus, la structure et le financement des recherches à long terme en Suisse auront lieu dans des ateliers fermés.

Au cours des symposiums, ateliers, présentations et séances de poster proposés les 25 et 26 août 2011, la conférence annuelle de Santé Publique Suisse se penchera plus particulièrement sur les maladies chroniques non transmissibles.

Le débat public de clôture (26 août 2011 à 15 heures) sera consacré au thème de la ville saine. L'urbanisme doit lui aussi faire face aux défis posés par les maladies chroniques. L'habitat densifié implique-til inévitablement bruit densifié, air pollué et accidents? Avons-nous besoin de cette «ville consommable»? A quoi ressemble la ville «en mouvement»? Des personnalités issues du monde politique, des administrations, du monde scientifique et du monde économique entameront un débat critique sous la direction d'Urs Steiger, journaliste scientifique et expert en aménagement du territoire et environnement. Il est possible d'assister au débat public gratuitement sans inscription. Bienvenue à Bâle!

1 Beaglehole R et al. for The Lancet NCD Action Group and the NCD Alliance. Priority actions for the noncommunicable disease crisis. Lancet. 2011;377: $1438-47$. 